\title{
The stellar populations in low excitation and high excitation radio galaxies
}

\author{
Michael B. Pracy ${ }^{1}$, John Ching ${ }^{1}$, Scott Croom $^{1}$ and Elaine M. Sadler ${ }^{1}$ \\ ${ }^{1}$ Sydney Institute for Astronomy, School of Physics, University of Sydney, NSW, 2006, \\ Australia \\ email: mpracy@physics.usyd.edu.au
}

\begin{abstract}
We have conducted deep optical spectroscopic follow up of a sample of radio galaxies with redshifts $z<0.7$. The spectra were used to construct robust sub-samples of low excitation and high excitation AGN and perform stellar population analysis via line indices and spectral fitting. While the high excitation objects have lower luminosity-weighted ages and lower metallicities than the low excitation galaxies, this can be explained by the different stellar mass distributions of the samples. When stellar mass is taken into account the age and metallicity distribution of both populations are consistent with the galaxy population as a whole.
\end{abstract}

Keywords. galaxies: active, galaxies: stellar content

\section{Introduction}

Radio mode feedback is commonly invoked as a mechanism to inhibit gas cooling and suppress star formation in galaxies and clusters of galaxies (e.g. Croton et al. 2006; McNamara \& Nulsen 2007). Radio jets produced from the accretion of material onto a supermassive black hole deposit energy into the surrounding gas, heating it and quenching star formation. The properties of the radio galaxy population indicate that two fundamentally different accretion modes are powering the emission (Hardcastle et al. 2007). In the standard cold-mode accretion, material is deposited onto the central super-massive black hole via a luminous accretion disk. This disk emits ionizing photons which give rise to both broad and narrow emission lines in the optical spectra (although the broad lines are obscured by a dusty torus unless the accretion disk is viewed face on) and X-ray emission via the inverse-Compton process.

However, there is a large population of radio galaxies that lack optical emission lines (e.g. Jackson \& Rawlings 1997; Best \& Heckman 2012), evidence for a luminous accretion disk or dusty torus (e.g. Whysong \& Antonucci 2004; Ogle et al. 2006) and are likely powered by radiatively inefficient accretion from a hot gas halo (e.g. Hardcastle et al. 2007). In the local Universe these Low Excitation Radio galaxies† (LERGs) outnumber the High Excitation Radio Galaxiesł (HERGs) at all but the highest radio luminosities. Hot mode accretion powered radio jets provide a natural feedback mechanism since the static hot gas atmosphere from which material cools and accretes is the same gas into which energy from the radio jets is deposited allowing a self regulating feedback cycle. In the cold mode the source of the accretion is independent of the hot gas with which the radio jet interacts. This allows large amounts of energy to be deposited into the intra-group or intra-cluster medium (Hardcastle et al. 2007) but there is no radio mode feedback cycle operating directly on the galaxy itself.

$\dagger$ Also referred to as 'radio mode' or 'hot mode'

$\ddagger$ Also referred to as 'quasar mode' or 'cold mode' 
It is well established that LERGs, those with weak or absent emission lines, are generally more massive, redder, have higher mass-to-light ratios and are of earlier morphological type than strong-lined AGN (e.g. Kauffmann et al. 2008; Best \& Heckman 2012). Kauffmann et al. (2008) found very little difference in the stellar populations between radio loud and radio quiet emission line AGN matched in stellar mass, redshift and velocity dispersion. However, the radio loud population does strongly prefer denser environments. Conversely, optically selected powerful AGN have significantly younger stellar populations than a matched sample without regard to their AGN status (Kauffmann et al. 2008). The presence of recent star formation in cold-mode AGN is qualitatively expected since both require the presence of cold gas. Best \& Heckman (2012) constructed a sample of HERGs and a sample of LERGs matched in redshift, stellar mass, black hole mass, and radio luminosity. They found the HERGs to have higher Eddington-scaled accretion rates, to be bluer, (marginally) smaller, less concentrated, and have lower $4000 \AA$ breaks than the LERGs.

While these studies have included large, robustly selected samples, and control samples, only simple diagnostics of the stellar populations have been used: such as the strength of the $4000 \AA$ break and broad-band colors. Both of these have a strong age-metallicity degeneracy. Here, we present spectroscopy of a sample of LERGs and HERGs. We use this to investigate the stellar populations of these objects out to $z=0.7 \mathrm{using}$ index measurements as well as full spectrum fitting.

\section{LERG and HERG samples}

The radio galaxy sample (Ching et al., in prep) was constructed by matching the Faint Images of the Radio Sky at Twenty-cm survey (Becker, White \& Helfand 1995) with the optical photometric catalogue of the Sloan Digital Sky Survey (SDSS). The catalogues were matched down to a flux limit of $\sim 1 \mathrm{mJy}$ in the radio catalogue and a limit of $i=21.5$ (SDSS $\mathrm{i}-$ band model mags) in the optical. The matching procedure was designed to include both point and extended radio sources. Follow up spectroscopy was obtained as part of the AAOmega-UKIDSS-SDSS (AUS) survey (Croom et al., in prep) of the stripe- 82 region of the SDSS. The data cover the wavelength range of $3700 \AA$ to $8800 \AA$ at a resolution of $\mathrm{R}=1300$. Typical exposure times per pointing were 6 hours.

We construct our LERG sample as radio galaxies with spectra having weak or no optical emission lines; defined here as spectra having the $\mathrm{H} \beta$ and $\mathrm{H} \alpha$ lines in absorption and insignificant emission in both the [OII] $\lambda 3727$ and [OIII] $\lambda 5007$ lines (equivalent width in emission of less than $2 \AA$ ). For the HERG sample we require radio galaxies possessing Seyfert 2 type spectra. We begin by constructing a sample which has all of the above lines in emission. These galaxies are then classified using standard line ratio diagnostics. For low redshift objects we used the Baldwin, Phillips \& Terlevich (1981, hereafter BPT) diagram. At redshifts greater than $z \sim 0.32$ the [NII] $\lambda 6583$ and $\mathrm{H} \alpha$ lines are shifted off the red edge of the AAOmega spectra and the BPT diagram is no longer available to us. These galaxies are classified using the 'blue diagram' (Lamareille 2010) which uses the $[\mathrm{OII}] \lambda 3726+\lambda 3729, \mathrm{H} \beta$ and $[\mathrm{OIII}] \lambda 5007$ lines and enables us to extend our spectral classification up to $\mathrm{z} \sim 0.7$. While there is overlap of different spectral classes in the 'blue diagram' the Seyfert 2 galaxies are robustly separated from the other spectral classes (Lamareille 2010) and it is therefore suitable for our purposes. Figure 1 shows the BPT diagram for the low redshift sample (left panel) and the blue diagram for the high redshift sample (right panel). Since the majority of the low redshift objects from the BPT diagram can also be placed on the blue diagram we can check the robustness of the blue diagram classification using these objects. While there is overlap of the other spectral types the 

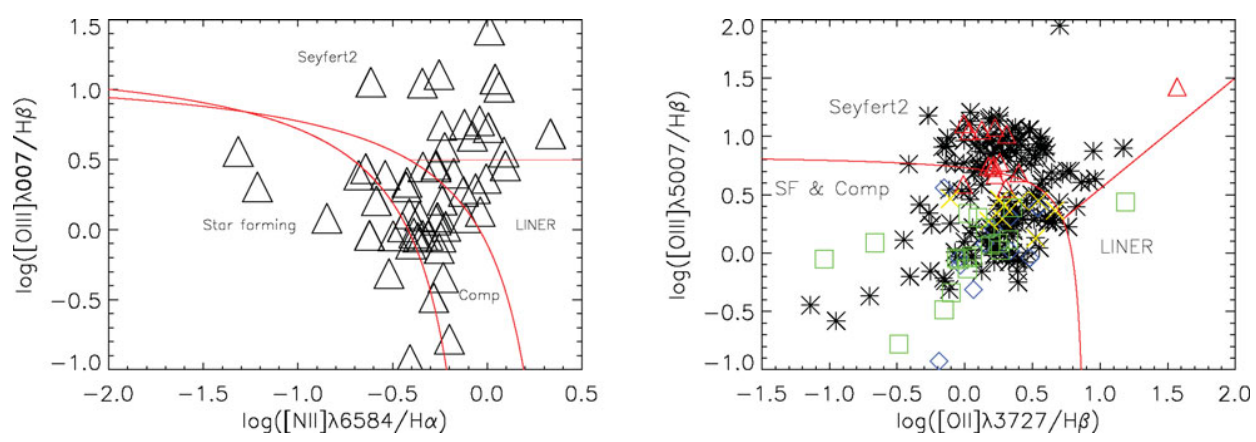

Figure 1. Left panel: The BPT diagram for objects with $z \lesssim 0.3$ with all diagnostic lines in emission. Right panel: The blue diagram (Lamareille 2010) for objects at $z \gtrsim 0.3$, where $\mathrm{H} \alpha$ and $[\mathrm{NII}] \lambda 6583$ are not available (black stars). The low redshift points are over-plotted coded by their position in the BPT diagram: red triangles are Seyfert 2s; yellow crosses are LINERS; green squares are composite objects; and blue diamonds are star forming.
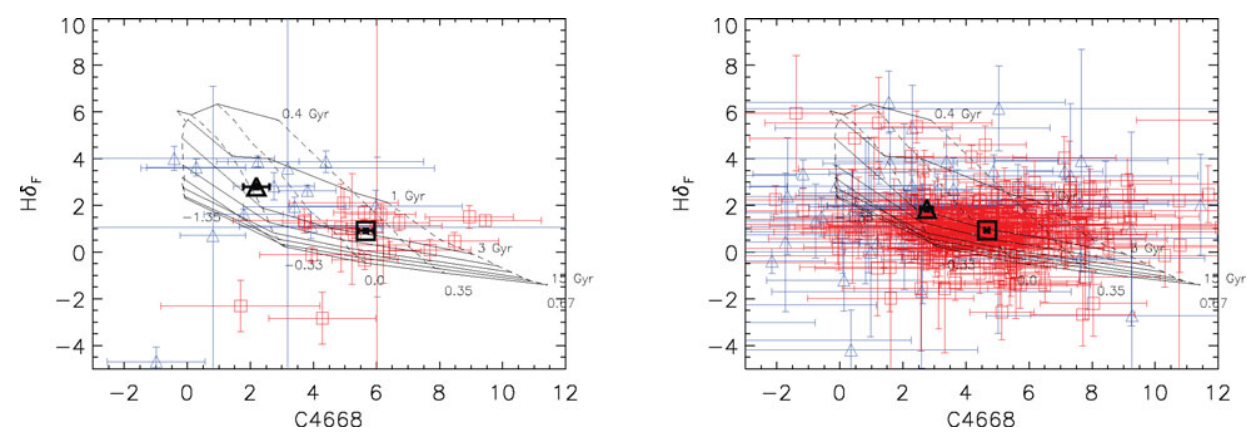

Figure 2. Example age-metallicity diagnostic diagrams for the low redshift, $z<0.3$, objects (left) and the high redshift sample (right). The LERGs are plotted as red squares and the HERGs as blue triangles. The robust mean of the distributions are shown as a black square and triangle, respectively. The model grids are derived from the stellar population models of Thomas et al. (2004).

Seyfert 2 galaxies (red triangle in Figure 2) are correctly defined on the blue diagram giving confidence in our classification at high redshift.

\section{Stellar populations}

We measured standard line indices in our spectra for the LERGs and HERGs. For the HERGs any emission was removed by fitting the spectra simultaneously with a set of single-age, single-metallicity stellar population models (Vazdekis et al. 2010), and emission line templates. An example line index diagram is shown in Figure 2 for the low redshift (left panel) and high redshift (right panel) samples. The HERGs have lower metallicities and younger stellar populations than the LERGs.

While there is a clear difference in the position of LERGs and HERGs in the agemetallicity diagnostics, it is not clear how much of this is the result of the populations having intrinsically different stellar masses. In Figure 3 we plot the luminosity-weighted ages and metallicities of the stellar populations derived from the stellar population fitting against stellar mass. The black contours show the distribution for the SDSS galaxies as a whole from Gallazzi et al. (2005). This demonstrates that the difference in age and metallicity between the LERGs and HERGs is consistent with being the result of 

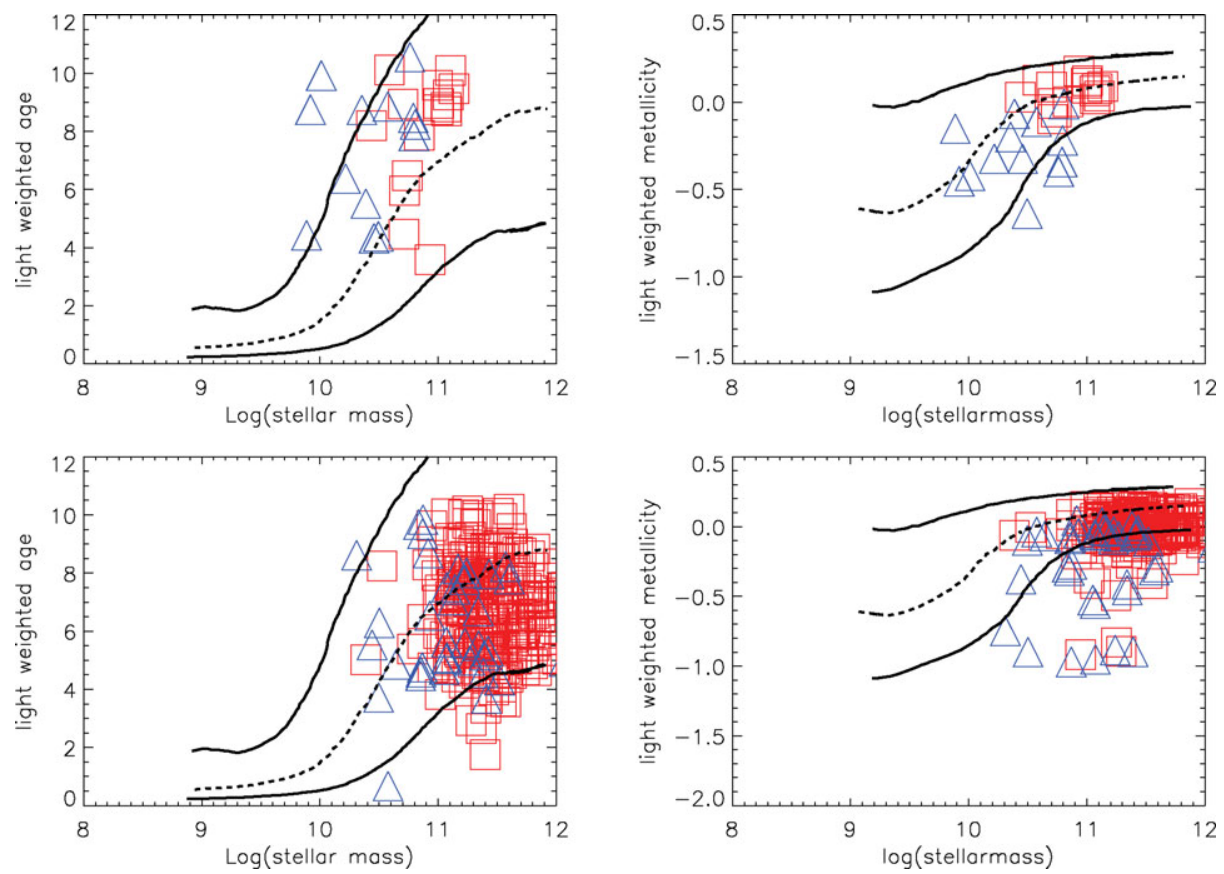

Figure 3. Top left: luminosity weighted age versus stellar mass for the $z<0.3$ objects. The HERGs (blue triangles) are generally lower stellar mass than the LERGs (red squares). Top right: luminosity weighted metallicity versus stellar mass for the $z<0.3$ objects. For comparison the black over-plotted locus is for the SDSS galaxies from Gallazzi et al. (2005). Bottom: same as top but for the $0.3<z<0.7$ galaxies.

differences in the stellar mass distributions between the samples. Also evident is redshift evolution to younger ages and lower metallicities at high redshift.

\section{References}

Baldwin, J. A., Phillips, M. M., \& Terlevich, R. 1981, PASP, 93, 5

Becker, R. H., White, R. L., \& Helfand, D. H. 1995, ApJ, 450, 559

Best, P. N. \& Heckman, T. M. 2012, MNRAS, 421, 1569

Ching, J., et al. 2013, in preparation

Croom, S. M., et al. 2013, in preparation

Croton, D. J., et al. 2006, MNRAS, 365, 11

Gallazzi, A., Charlot, S., Brinchmann, J., \& White, S. D. M., Tremonti C. 2005, MNRAS, 362, 41

Hardcastle, M. J., Evans, D. A., \& Croston, J. H. 2007, MNRAS, 376, 1849

Jackson, N. \& Rawlings, S. 1997, MNRAS, 286, 241

Kauffmann, G., Heckman, T. M., \& Best, P. N. 2008, MNRAS, 384, 953

Lamareille, F. 2010, A\& $A, 509,53$

McNamara, B. R. \& Nulsen, P. E. J. 2007, ARA\&A, 45, 117

Ogle, P., Whysong, D., \& Antonucci, R. 2006, ApJ, 647, 161

Thomas, D., Maraston, C., \& Korn, A. 2004, MNRAS, 351, L19

Vazdekis, A., et al. 2010, MNRAS, 404, 1639

Whysong, D. \& Antonucci, R. 2004, ApJ, 602, 116 